\title{
A Death Due to Neurobrucellosis - Linfen City, Shanxi Province, China, May 2021
}

\author{
Yufeng Du'; Hongxia Yang'; Hai Jiango,; Xiufang $\mathrm{Du}^{1, *,}$
}

On May 8, 2021, a death due to brucellosis was reported to the Shanxi CDC. An epidemiological investigation was conducted to determine the cause of death.

The patient, a 41-year-old man engaged in mining, was brought to the emergency room of Xinjiang Military General Hospital on March 13, 2021, with complaints of gradual-onset fever and headache. $\mathrm{He}$ was admitted for diagnostic workup. Two days later, he developed convulsions and altered consciousness and was transferred to the intensive care unit. After a week, the patient again developed high-grade fever and delirium, with his temperature rising to $39.5^{\circ} \mathrm{C}$. The fever was not associated with chills. There was no history of exposure to toxic substances. The patient's wife reported that he had consumed roasted mutton. Blood culture and next-generation sequencing of cerebrospinal fluid (CSF) were both positive for Brucella. Based on clinical manifestations, epidemiological history, and etiological test results, the patient was diagnosed with neurobrucellosis.

He was transferred to Xinjiang Medical University Affiliated Hospital on March 25. He was treated with intravenous antibiotics (ceftriaxone, rifampicin, and doxycycline) and sodium valproate. However, fever, headache, and disturbance of consciousness persisted. Head computerized tomography (CT) revealed large brain abscesses and cerebral herniation. Surgical treatment was advised, but the patient's family refused surgery and asked for transfer back to the local hospital. On April 1, the patient was transferred to Linfen Infectious Disease Hospital, Shanxi Province. Repeat CT scan showed enlargement of the brain abscesses. Multiple large, annular low-density shadows were seen in the left temporal lobe, frontal lobe, and basal ganglia region, with a fluid plane visible in the latter. The left lateral ventricle was compressed, and the midline was displaced to the right (Figure 1). At 20:30 on April 23, physical examination showed bilateral dilated pupils, with sluggish response to light, and absent orbital pressure reflex. On April 25, the patient died.

Human brucellosis is a multisystem disease that can present with a broad spectrum of clinical manifestations and complications. Death is usually due to endocarditis or neurobrucellosis. Neurobrucellosis, which may occur at any stage of the disease, develops in $<5 \%$ of patients with Brucella infection and presents with diverse neurological symptoms and signs (1-3). Standard first-line treatment is with doxycycline, rifampicin, and third-generation cephalosporins for at least 6 weeks. Prognosis varies according to clinical manifestations (4). The diagnosis of neurobrucellosis is based on clinical and radiological features and serological tests. Underdiagnosis of neurobrucellosis may be due to poor awareness among health workers
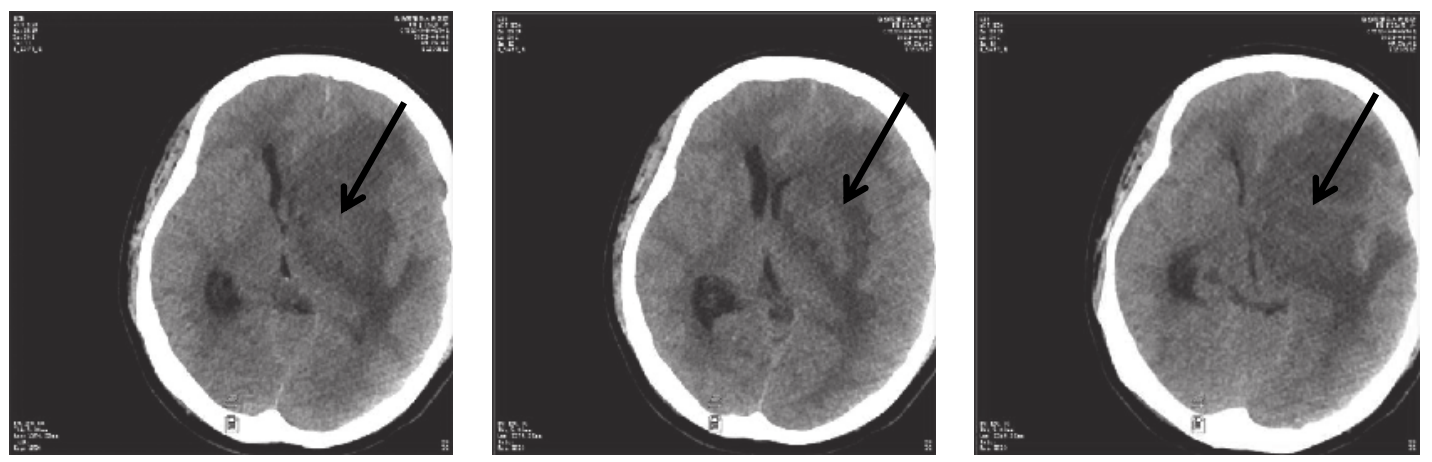

FIGURE 1. The progression of the brain abscess for the patient by computerized tomography (CT) - Linfen, Shanxi, China, 2021. 
and lack of diagnostic facilities.

There are few reports of neurobrucellosis from China (5). In this paper, delayed diagnosis and extra referrals likely factored into the serious outcome. The present case report highlights the importance of early diagnosis of neurobrucellosis in the laboratory. We recommend the following measures: 1) establishing rapid detection methods, such as polymerase chain reaction, for early diagnosis; 2) raising the awareness of healthcare workers, especially in the department of neurology; and 3) improving multidisciplinary support for case management.

Conflicts of interest: No conflicts of interest declared.

Funding: National Key R\&D Program of China (2020YFA0907101).

\section{doi: $10.46234 / \mathrm{ccdcw} 2021.238$}

* Corresponding authors: Hai Jiang, jianghai@icdc.cn; Xiufang Du, crduxiufang@163.com.

\footnotetext{
${ }^{1}$ Linfen Third People's Hospital, Linfen, Shanxi, China; ${ }^{2}$ Shanxi
}

Provincial Centre for Disease Control and Prevention, Taiyuan, Shanxi, China; ${ }^{3}$ State Key Laboratory for Infectious Disease Prevention and Control, Collaborative Innovation Center for Diagnosis and Treatment of Infectious Diseases, National Institute for Communicable Disease Control and Prevention, Chinese Center for Disease Control and Prevention, Beijing, China.

Submitted: September 03, 2021; Accepted: November 04, 2021

\section{REFERENCES}

1. Guven T, Ugurlu K, Ergonul O, Celikbas AK, Gok SE, Comoglu S, et al. Neurobrucellosis: clinical and diagnostic features. Clin Infect Dis 2013;56(10):1407 - 12. http://dx.doi.org/10.1093/cid/cit072.

2. McLean DR, Russell N, Khan MY. Neurobrucellosis: clinical and therapeutic features. Clin Infect Dis 1992;15(4):582 - 90. http://dx.doi. org/10.1093/clind/15.4.582.

3. Zheng N, Wang W, Zhang JT, Cao Y, Shao L, Jiang JJ, et al. Neurobrucellosis. Int J Neurosci 2018;128(1):55 - 62. http://dx.doi.org/ 10.1080/00207454.2017.1363747.

4. Ceran N, Turkoglu R, Erdem I, Inan A, Engin D, Tireli H, et al. Neurobrucellosis: clinical, diagnostic, therapeutic features and outcome. Unusual clinical presentations in an endemic region. Braz J Infect Dis 2011;15(1):52 - 9. http://dx.doi.org/10.1016/S1413-8670(11)70140-4.

5. Wu TY. Clinicopathological report of brucellosis complicated with meningoencephalitis, diabetes insipidus and cirrhosis. Chin J Intern Med 1961;9(7):449. (In Chinese). 